Article

\title{
The Phase Space Elementary Cell in Classical and Generalized Statistics
}

\author{
Piero Quarati ${ }^{1,2, *}$ and Marcello Lissia ${ }^{2}$ \\ ${ }^{1}$ DISAT, Politecnico di Torino, C.so Duca degli Abruzzi 24, Torino I-10129, Italy \\ ${ }^{2}$ Istituto Nazionale di Fisica Nucleare, Sezione di Cagliari, Monserrato I-09042, Italy; \\ E-Mail: marcello.lissia@ca.infn.it \\ * Author to whom correspondence should be addressed; E-Mail: pierino.quarati@ca.infn.it; \\ Tel.: +39-0706754899; Fax: +39-070510212.
}

Received: 05 September 2013; in revised form: 25 September 2013/ Accepted: 27 September 2013 /

Published: 15 October 2013

\begin{abstract}
In the past, the phase-space elementary cell of a non-quantized system was set equal to the third power of the Planck constant; in fact, it is not a necessary assumption. We discuss how the phase space volume, the number of states and the elementary-cell volume of a system of non-interacting $N$ particles, changes when an interaction is switched on and the system becomes or evolves to a system of correlated non-Boltzmann particles and derives the appropriate expressions. Even if we assume that nowadays the volume of the elementary cell is equal to the cube of the Planck constant, $h^{3}$, at least for quantum systems, we show that there is a correspondence between different values of $h$ in the past, with important and, in principle, measurable cosmological and astrophysical consequences, and systems with an effective smaller (or even larger) phase-space volume described by non-extensive generalized statistics.
\end{abstract}

Keywords: classical statistical mechanics; thermodynamics

\section{Introduction}

In a microcanonical formulation, the entropy is proportional to the logarithm of the number of states, $W$, accessible to the systems.

In quantum mechanics, we can count the number of microstates $W$ corresponding to a microstate of total energy, $E$. This number of microstates $W$ is often called the multiplicity of the microstate. 
In classical mechanics, the state of an $N$-particle system is represented in a $6 N$ dimensional coordinate-momentum space ( $\Gamma$ space). Boltzmann solved the problem of infinities when counting the multiplicity of continuous states by dividing the phase-space in elementary cells, whose volume $\Delta \Omega$ remains arbitrary in classical mechanics.

The advent of quantum mechanics provided the framework to determine the volume of the smallest elementary cell, which is given by the cube of the Planck constant: $h^{3}$. This lower bound to the volume of the elementary cell is directly connected to the uncertainty principle.

In classical statistical mechanics, the arbitrary volume of the elementary cell does not affect physical quantities. In fact, as long as the entropy of the system is proportional to the logarithm of the number of states: $W=\Omega /(\Delta \Omega)^{N}$

$$
S=k \ln \frac{\Omega}{(\Delta \Omega)^{N} N !}
$$

where $\Omega$ is the volume of the phase space accessible to the system, entropy differences:

$$
\Delta S=S(A)-S(B)
$$

do not depend on the volume of the elementary cell $\Delta \Omega$, and therefore, all physical quantities that depend on entropy differentials, such as the heat capacity:

$$
C_{V}=T\left(\frac{\partial S}{\partial T}\right)_{(V, N)}
$$

or the pressure:

$$
P=T\left(\frac{\partial S}{\partial V}\right)_{(E, N)}
$$

are similarly independent of the choice of the elementary cell volume.

However, the chemical potential:

$$
\mu=-T\left(\frac{\partial S}{\partial N}\right)_{(E, V)}
$$

does depend on $\Delta \Omega$. Therefore, the tendency of particles to diffuse along the gradient of densities or, more generally, of chemical potential appears to be affected by the elementary volume of the the phase space. Furthermore, the elementary cell enters also in the Sackur-Tetrode entropy of a mono-atomic classical ideal gas:

$$
\frac{S}{k N}=\frac{5}{2}+\ln \left[\frac{V}{N}\left(\frac{E}{N}\right)^{3 / 2} \frac{m}{3 \pi h^{2}}\right]
$$

where $E$ is the internal energy of the gas and $m$ the mass of the particles of the system. In fact, this entropy incorporates quantum effects through, for instance, the thermal wave length $\sqrt{3 \pi h^{2} N /(m E)}$.

Up to now, we have considered classical systems composed of non-interacting classical particles or of particles subjected to short-range forces that do not alter the basic one-particle structure of the excitations and that can be absorbed in a few effective parameters. This kind of systems can be described by the Maxwell-Boltzmann (MB) statistics, and their excitation distribution is obtained by the maximization method. 
The number of different microstates $w$ accessible to a system of $N$ identical particles is obtained by dividing by $N$ ! the number of microstates $W$ of a corresponding system of distinguishable particles:

$$
w=\frac{W}{N !}
$$

which is greatly reduced with respect to $W$. According to Gibbs, all microstates in this microcanonical ensemble have equal probabilities.

In classical statistical mechanics, the statement $(\Delta \Omega)^{N}=h^{3 N}$ is justified considering the product of an $N$ independent quantum one-particle system or from the pure dimensional point of view: the Planck constant has dimensions of the two-dimensional phase-space volume $x \times p$, and each particle occupies a volume $\Delta \Omega=h^{3}$. A possible dimensionless factor cannot be excluded classically.

In conclusion, in classical discrete and continuum statistical mechanics, the volume of an elementary cell is not determined a priori; it is dimensionless in units of the cube of the Planck constant and could span a large set of values. The choice that the cell has a volume exactly equal to $h^{3}$ is plausible, but only the experiment could possibly determine this constant in classical mechanics.

There exist complex natural and artificial systems characterized by long-range space-time correlations whose dynamics cover only a multi-fractal subset of the whole phase space and develop non-Gaussian correlations, at least for practical finite equilibration times. These kinds of systems can be effectively described by generalized statistical mechanics that are a continuous deformation of the usual one [1,2]. A particularly simple such generalized approach is the one that uses the non-extensive Tsallis entropy $[3,4]$ in its continuous and quantum versions. We have already studied modifications of the phase-space elementary cell in the context of non-extensive statistical mechanics [5]; this work develops such an approach on a more general basis.

Recently, S. Abe [6] used this choice $\Delta \Omega=h^{3}$, but only for classical discrete systems. He argues that variation of entropy or other measurable quantities in non-extensive systems do not depend on $h^{3}$ only in discrete systems. On the contrary, entropy or other measurable quantities in continuum non-extensive systems depend on $h^{3}$ against the hypothesis that the systems are classical. We shall elaborate on this point in Section 3.

In this paper, we show how the phase-space volume can be deformed and how the non-extensive expression of the number of states changes from the one of an ideal non-interacting particle system. We obtain first the value of the deformed phase space volume. Then, we deduce the elementary cell volume as a function of the entropic deformation parameter.

Even quantum systems, which appear to have a lower bound to the phase-space volume, due to the uncertainty principle, could have an effective volume of the elementary cell slightly smaller than $h^{3}$ in non-Boltzmann systems with a fractal measure of the volume. In addition, there exists the possibility that the Planck constant and, therefore, the volume $h^{3}$ were different in the past. Both possibilities would have measurable cosmological and astrophysical consequences. Entropic uncertainty relations are also considered.

\section{The Deformation of Phase Space Volume}

The very large number of microstates makes both the phase-space and elementary-cell volume practically insensitive to small variations of the distribution function. In fact, the likelihood of deviations 
is exponentially small, with the exponent proportional to the large number of degrees of freedom. However interactions can create strong correlations that strongly reduce the effective number of degrees of freedom relevant at finite time-scales.

Since the equilibrium (maximum) value, $W_{0}$, is much larger than the values of the number of microstates $W$ associated with distributions even slightly different from equilibrium, one usually identifies the state of maximum probability with the real state that is experimentally measured (Sommerfeld [7], p. 218). However, we will show how going from an extensive classical system to a non-extensive one, i.e., from a pure MB distribution to distributions with power-law tails, small changes of the phase-space volume can influence the description of the system.

The effect on $\Omega$ of variations from the Maxwellian distribution function $n_{i o}$ of the $i$-th state can be found in the paper by Bohm and Schutzer [8]. In the book of Diu et al. [9], it is shown that in classical physics, the elementary cell is the Planck constant by comparing the sequence of the admissible states to the sequence of states in quantum mechanics.

Long-range interactions, correlations, memory effects, a fractal space, elementary length or discrete time are some of the physical reasons systems could not be consistently described by MB distributions. Such systems can often be better described by generalized statistics, as developed in the last few years. In fact, one might also view these effective descriptions as non-equilibrium metastable states: if the lifetime is long enough, the final thermodynamical equilibrium is not experimentally relevant.

For the sake of simplicity, we discuss only the so-called $q$-statistics [3,4]. However, similar conclusions could be reached with other power-law deformations of the MB statistics, such as the $\kappa$-statistics $[10,11]$, which offers a mathematically attractive context, symmetry properties, and interesting relations with the formalism of relativity.

Criticisms to the use of non-extensive, generalized statistics can be found, for instance, in works by Gross [12]. In Shalizi [13], for instance, one can find criticisms of the use of approaches that adopt deformed, non-extensive or generalized statistics.

In the following, we show how the phase-space volume varies for small deviations from the standard distribution function using the specific formalism of the generalized $q$-statistics.

The phase space volume for a system of $N$ non-interacting Boltzmann particles (or molecules) is defined by:

$$
\Omega_{0}=\frac{N !}{n_{10} ! n_{20} ! \cdots} \frac{1}{N !}\left(\Delta \Omega_{0}\right)^{N}
$$

for interacting non-Boltzmann particles, we define:

$$
\Omega_{q}=\frac{N !}{n_{1 q} ! n_{2 q} ! \cdots} \frac{1}{N !}\left(\Delta \Omega_{q}\right)^{N}
$$

where $n_{i 0}$ and $n_{i q}$ are, respectively, the standard and deformed distributions. The number of accessible states is:

$$
W_{0}=\frac{N !}{n_{10} ! n_{20} ! \cdots}=N ! \frac{\Omega_{0}}{\left(\Delta \Omega_{0}\right)^{N}}
$$

and:

$$
W_{q}=\frac{N !}{n_{1 q} ! n_{2 q} ! \cdots}=N ! \frac{\Omega_{q}}{\left(\Delta \Omega_{q}\right)^{N}}
$$

We are interested in small deviations from the standard distributions and define: $\delta n_{i}=n_{i q}-n_{i 0}$. 
Using the Stirling formula, $\ln W_{q}=\ln (N !)-\sum_{i} \ln \left(n_{i q} !\right)$ can be expanded around the value $W_{0}\left(n_{i 0}\right)$ that maximizes the phase volume $\Omega_{0}$ at the MB distribution, which is expressed as:

$$
n_{i 0}=A_{M} e^{-a_{i}} \text { with } a_{i} \equiv \beta \epsilon_{i}
$$

where

$$
A_{M}=\frac{N}{V}\left(\frac{\beta}{2 \pi m}\right)^{3 / 2} \Delta \Omega_{0}
$$

and the normalization is such that $\sum_{i} n_{i 0}=N$. In this expansion:

$$
\ln W_{q}=\left(N \ln N-\sum_{i} n_{i 0} \ln n_{i 0}\right)-\sum_{i}\left(1+\ln n_{i 0}\right) \delta n_{i}-\frac{1}{2} \sum_{i} \frac{\delta n_{i}^{2}}{n_{i 0}}+\cdots
$$

terms linear in $\delta n_{i}$ vanish, because of the maximum conditions, and retaining the leading contribution, the explicit expressions of $W_{q}$ and $\Omega_{q}$ for a gas that deviates only slightly from the MB distribution, assuming for the moment an unchanged elementary cell, are:

$$
W_{q}=W_{0} \exp \left(-\frac{1}{2} \sum_{i} \frac{\delta n_{i}^{2}}{n_{i 0}}\right)
$$

and:

$$
\Omega_{q}=\Omega_{0} \exp \left(-\frac{1}{2} \sum_{i} \frac{\delta n_{i}^{2}}{n_{i 0}}\right)
$$

The deformed distribution is:

$$
n_{i q}=A_{M} A_{q} e^{-\beta \epsilon-(1 / 2)(1-q)(\beta \epsilon)^{2}}
$$

with $\sum_{i} n_{i q}=N$ where:

$$
A_{q}=1+\frac{15}{8}(1-q)+o(1-q)^{2}
$$

For $q \approx 1$, we have:

$$
\begin{aligned}
n_{i q}=n_{i 0}\left(1+\frac{15}{8}(1-q)-\frac{(1-q)}{2}\left(\beta \epsilon_{i}\right)^{2}\right) \\
\sum_{i} \frac{\left(\delta n_{i}\right)^{2}}{n_{i 0}}=\frac{(1-q)^{2}}{4} \sum_{i} n_{i 0}\left[\frac{15}{4}-\left(\beta \epsilon_{i}\right)^{2}\right]^{2} \\
=N \frac{(1-q)^{2}}{4}\left[\left(\frac{15}{4}\right)^{2}-\frac{15}{2}\left\langle\left(\beta \epsilon_{i}\right)^{2}\right\rangle+\left\langle\left(\beta \epsilon_{i}\right)^{4}\right\rangle\right] \\
=N \frac{(1-q)^{2}}{4}\left[\left(\frac{15}{4}\right)^{2}-\frac{15}{2} \frac{15}{4}+\frac{945}{16}\right]=\frac{45}{4} N(1-q)^{2}
\end{aligned}
$$

where in the preceding equations, we have calculated the average values with the unperturbed distribution.

Therefore, we obtain:

$$
W_{q}=W_{0} e^{-(45 / 8) N(1-q)^{2}}
$$


From $W_{q} / W_{0}=\left(\Omega_{q} / \Omega_{0}\right)\left(\Delta \Omega_{0} / \Delta \Omega_{q}\right)^{N}$ (see, for instance, Equations (10) and (11)), this change can be interpreted microscopically as a change of an elementary cell leaving the phase-space volume unchanged:

$$
\Delta \Omega_{q}=\Delta \Omega_{0} e^{(45 / 8)(1-q)^{2}}
$$

Because the correction factor to $W_{q}$ depends on $(q-1)^{2}, \Delta \Omega_{q}$ is always larger than $\Delta \Omega_{0}$. However, if we had assumed that the number of accessible states were independent of $q$, $\Omega_{q}$ and $\left(\Delta \Omega_{q}\right)^{N}$ would change in the same way, and one could obtain an elementary cell smaller than $\Delta \Omega_{0}$; in particular, $\Delta \Omega_{q}=\Delta \Omega_{0} \exp \left(-(45 / 8)(1-q)^{2}\right.$. Here, we just remark that $W_{0}$ and $W_{q}$ have different values only to the second order in an expansion in $(1-q)$. In the definition of the standard entropy, we use $W_{0}$, while in the definition of the $q$-entropy, we should use the $q$-logarithm of $W_{q}$. However, the leading linear correction comes only from the $q$-logarithm, and one can use $W_{0}$.

\section{Classical Volume Cell, Continuous States and Non-Maxwellian Statistics}

When computing the entropy of a classical system with states parameterized by continuous parameters, coarse graining, i.e. a finite cell size, overcomes the difficulties of defining the correct measure of probability densities. Abe [6] argues that generalized statistical mechanics with non-logarithmic entropies can be applied only to physical systems with discrete degrees of freedom. In fact, the elementary volume necessary to regularize the sum over states in continuous Hamiltonian systems does not disappear in the final results for non-additive entropic functions: if the elementary cell is assumed to be $h^{3}$ from the underling quantized formulation, classical results would contain the quantum constant $h^{3}$. This reasoning spurred an interesting discussion [14,15] between Abe and Andresen.

We remark that a discrete cell can appear also in classical mechanics, for instance, due to effective coarse graining with a volume not necessarily equal to $h^{3}$. In addition, the same constant $h$ can have an interpretation also in a classical context [16-18].

We conclude that both continuous and discrete classical systems can contain a phase space elementary cell that in classical physics can have any value: in particular, its volume can be $h^{3}$. Therefore, both continuous and discrete system can be described by generalized statistical mechanics that permit elementary cells with a volume different from $h^{3}$.

\section{The Generalized Number of States and the Generalized Elementary Cell Volume}

We consider the standard classical phase space $\Omega_{0}$, which is maximum when the global thermodynamical equilibrium distribution $n_{i 0}$ is the MB distribution for the $i$-th state. The elementary phase space cell is $\Delta \Omega_{0}$, and $N$ is the number of particles of the system.

Let us define the dimensionless quantity:

$$
M_{0}^{N} \equiv \frac{\Omega_{0}}{\left(\Delta \Omega_{0}\right)^{N}}=\frac{W_{0}}{N !}=w_{0}
$$

which is the measure of the $3 D$ phase-space volume relative to the volume of the one-particle cell $\Delta \Omega_{0}$ and where $w_{0}$ is the number of microstates. 
Analogously in the $q$-deformed formalism, we define the $q$-volume $\Omega_{q}$ and the $q$ elementary cell $\Delta \Omega_{q}$ and introduce the dimensionless measure:

$$
M_{q}^{\otimes_{q} N}=\frac{\Omega_{q}}{\left(\Delta \Omega_{q}\right)^{N}}=\frac{W_{q}}{N !}=w_{q}
$$

where we have used the $q$-power (see, for instance, [19]):

$$
M_{q}^{\otimes_{q} N}=\left[N M_{q}^{(1-q)}-N+1\right]^{1 /(1-q)}
$$

The expansion of $M_{q}^{\otimes_{q} N}$ for $N \gg 1$ around the value $q=1$ (see Appendix A for details) gives:

$$
\begin{aligned}
M_{q}^{\otimes_{q} N} & =M_{q}^{N}\left[1+\frac{q-1}{2} N^{2}\left(\ln M_{q}\right)^{2}\right] \\
& =\frac{W_{q}}{N !} \times\left[1+\frac{q-1}{2}\left(\ln \frac{W_{0}}{N !}\right)^{2}\right]
\end{aligned}
$$

where, inside the logarithm, we have used $M_{q}=M_{0}$ to the order in $1-q$ considered. If we divide the above equation by the corresponding equation with the index of zero $(q=1)$, we find:

$$
\frac{M_{q}^{\otimes_{q} N}}{M_{0}^{N}}=\frac{W_{q}}{W_{0}} \times\left[1+\frac{q-1}{2}\left(\ln \frac{W_{0}}{N !}\right)^{2}\right]=\left(\frac{\Omega_{q}}{\Omega_{0}}\right)\left(\frac{\Delta \Omega_{0}}{\Delta \Omega_{q}}\right)^{N}
$$

where the last equality derives from definitions (23) and (24) of $M_{0}^{N}$ and $M_{q}^{\otimes_{q} N}$. Therefore:

$$
\Delta \Omega_{q}=\Delta \Omega_{0}\left(\frac{W_{q}}{W_{0}}\right)^{-1 / N}\left[1+\frac{q-1}{2}\left(\ln \frac{W_{0}}{N !}\right)^{2}\right]^{-1 / N}\left(\frac{\Omega_{q}}{\Omega_{0}}\right)^{1 / N}
$$

Inserting the deformed expression of $W_{q}$, as derived in Equation (21), and ascribing the whole dependence on $q$ to the micro-physics, i.e. $\Omega_{q}=\Omega_{0}$, as it appears reasonable if the thermodynamical limit exists, we obtain, finally:

$$
\Delta \Omega_{q}=\Delta \Omega_{0} e^{(45 / 8)(q-1)^{2}} \times\left[1+\frac{q-1}{2}\left(\ln \frac{W_{0}}{N !}\right)^{2}\right]^{-1 / N} .
$$

The expression of the $q$-deformed elementary cell is made of two factors: the first is the elementary cell derived without the use of $q$-algebra (see Equation (22)); the second represents the correction due to the $q$-algebra. Note that the last relation shows how $\Delta \Omega_{q}$ can be smaller or larger than $\Delta \Omega_{0}$, depending on the value of $q$.

In Appendix B, we also consider the corresponding entropy with a few numerical examples.

\section{Entropic Uncertainty Relations and Elementary Cell}

The above sections show the link between elementary-cell volume and the uncertainty principle. It is well known that generalizations and extensions of the original uncertainty relations of Heisenberg have been proposed involving Shannon, Rényi and, later, Tsallis entropy. To be more clear: quantum 
mechanical uncertainty relations for momentum and position were expressed as inequalities in terms of Shannon, Rényi or Tsallis entropy.

Uncertainty phase space volume is related to the phase-space elementary cell that therefore can be expressed in terms of Shannon, Rényi and Tsallis entropy, giving us the elementary volume for these generalized statistics [20-27].

Let us introduce the experimental phase-space elementary cell volume $\Delta \Omega_{E}$ as the volume of phase space determined by the resolution of the measuring instruments. On the other hand, the elementary cell volume, as discussed in the previous sections, is related to the Heisenberg uncertainty principle and does not depend on the accuracy of our measuring instruments.

In the Shannon information formalism, the entropy is:

$$
H=-\sum_{k} p_{k} \ln p_{k}
$$

where $p_{k}$ is the dimensionless position distribution for the $k$ state $\left(\kappa_{B}=1\right)$. The quantity, $H$, is the position for Shannon. Analogously, $H$ can also be expressed for momentum.

In the Rényi framework [23], position and momentum entropy have the expressions:

$$
\begin{aligned}
H_{\alpha}^{x} & =\frac{1}{\alpha} \ln \sum_{k} p_{k}^{\alpha} \\
H_{\beta}^{p} & =\frac{1}{\beta} \ln \sum_{k} p_{k}^{\beta}
\end{aligned}
$$

where $H_{\alpha}^{x}$ and $H_{\beta}^{p}$ represent the uncertainty in position and momentum for Rényi statistics in the quantum world. When the entropic parameter $\alpha$ or $\beta$ goes to one, one recovers the Shannon entropy.

Shannon connected the content of the measure of information with a probability distribution, inserting the set of probabilities obtained from quantum mechanics into the information entropy.

We define the dimensionless relative volume of the cell: $\omega_{S}$

$$
\omega_{S}=\frac{\Delta \Omega_{E}}{\Delta \Omega_{0}}=\left(\frac{\delta x \delta p}{h}\right)^{3}
$$

This quantity decreases as we enter into the quantum regime. In the Shannon frame, we have identified $h^{3}$ with the standard elementary cell $\Delta \Omega_{0}$.

From the entropic uncertainty relations, we can give analytical expressions of $\omega$ in the Shannon, Rényi and Tsallis statistics.

In quantum mechanics and in information theory, the entropic uncertainty is defined as the sum of temporal and spectral entropies. The uncertainty principle of Heisenberg can be expressed as a lower bound on the sum of these entropies. This is much stronger than the standard statement of the uncertainty Heisenberg principle in terms of the product of standard deviations.

The Shannon entropic uncertainty relation can be written: as [23]

$$
H^{x}+H^{p} \geq 1-\ln 2-\frac{1}{3} \ln \omega_{S}
$$

By inverting the above relation, we obtain:

$$
\omega_{S} \geq e^{3-\ln 8} e^{-3\left(H^{x}+H^{p}\right)}
$$


The lower quantum mechanics bound on $\left(H^{x}+H^{p}\right)$ is found for $\omega=1:\left(H^{x}+H^{p}\right) \geq 1-\ln 2 \approx 0.307$. Corresponding to $\omega<1(>1)$, one has lower bounds greater (smaller) than $1-\ln 2$.

Let us report the following limiting cases (these limits are unphysical, because they violate the Heisenberg principle):

- if $\Delta \Omega_{E}$ goes to zero, then $\left(H^{x}+H^{p}\right)$ goes to infinity (complete lack of information);

- if $\left(H^{x}+H^{p}\right)=0$ (perfect information), then $\omega_{S}=\exp (3-\ln 8)$.

The Rényi entropic relation is:

$$
H_{\alpha}^{x}+H_{\beta}^{p} \geq-\frac{1}{2}\left(\frac{\ln \alpha}{1-\alpha}+\frac{\ln \beta}{1-\beta}\right)-\ln 2-\frac{1}{3} \ln \omega_{R}
$$

where $\omega_{R}=\Delta \Omega_{E} / \Delta \Omega_{R}, \Delta \Omega_{R}$ is the elementary cell in the Rényi frame, and the entropic parameters must verify $\alpha^{-1}+\beta^{-1}=2$. Therefore, from Equation (7) of [23], we derive:

$$
\omega_{R} \geq \frac{1}{8} e^{-3\left(H_{\alpha}^{x}+H_{\beta}^{p}\right)} e^{-\frac{3}{2}\left(\frac{\ln \alpha}{1-\alpha}+\frac{\ln \beta}{1-\beta}\right)}
$$

If the same measure of uncertainty is used for both variables, position and momentum, we obtain from Equation (46) of [23]:

$$
\omega_{\hat{R}} \geq \frac{1}{8} e^{-3\left(H_{\beta}^{x}+H_{\beta}^{p}\right)} \frac{1}{\beta^{3}}(2 \beta-1)^{(3 / 2-3 \beta) /(1-\beta)}
$$

with $1 / 2 \leq \beta \leq 1$.

The cell $\Delta \Omega_{R}$ can easily be derived in terms of the sum of position and momentum uncertainty and of the experimental phase-space elementary cell volume.

A detailed analysis in terms of the phase-space elementary cell needs to be performed. At the moment, we say that the volume of the elementary cell depends on the effective statistics relevant for the specific physical system, and its value in units of $h^{3}$ can be smaller than one; in some cases, this effective volume can be even much bigger than one. In addition, the Planck constant, and, therefore, the fine-structure constant $\hbar c / e^{2} \approx 137.036$, could have had values different from the one presently measured.

Existing generalizations of the uncertainty principle do not make reference to the implications for the volume of the elementary cell in quantum dynamics. Parallel modifications of the elementary cell can be studied within classical dynamics, where the scale $h^{3}$ is not derived from an uncertainty principle. For instance T. H. Boyer interprets $h$ as the scale of random classical zero-point radiation that is one of the solutions of the Maxwell equations [16-18].

We stress the important relation between non-extensive statistics and modifications of the elementary volume of phase space.

In the following, we summarize uncertainty entropic relations derived from Bialynicki-Birula [23] and Wilk $[24,26]$ using also the condition of the non-negativity of momentum entropy and position entropy and of the right-hand sides of inequalities (7) and (46) of [23] and (19) and (20) of [24]

For Shannon, we have:

$$
\frac{2}{e} e^{\left(H^{x}+H^{p}\right)} \geq \frac{\Delta \Omega_{0}^{1 / 3}}{\delta x \delta p} \geq \frac{2}{e}
$$


For Rényi, we have:

from Equation (7) of [23] if $\alpha \neq \beta$ with $\alpha \geq 1$ and $\beta \leq 1$ :

$$
2 e^{\frac{1}{2}\left(\frac{\ln \alpha}{1-\alpha}+\frac{\ln \beta}{1-\beta}\right)} e^{\left(H_{\alpha}^{x}+H_{\beta}^{p}\right)} \geq \frac{\Delta \Omega_{R}^{1 / 3}}{\delta x \delta p} \geq 2 e^{\frac{1}{2}\left(\frac{\ln \alpha}{1-\alpha}+\frac{\ln \beta}{1-\beta}\right)}
$$

where $1 / \alpha+1 / \beta=2$;

from Equation (46) of [23], if $\alpha=\beta$ :

$$
2 \beta(2 \beta-1)^{\frac{\beta-1 / 2}{1-\beta}} e^{H_{\beta}^{x}+H_{\beta}^{p}} \geq \frac{\Delta \Omega_{\hat{R}}^{1 / 3}}{\delta x \delta p} \geq 2 \beta(2 \beta-1)^{\frac{\beta-1 / 2}{1-\beta}}
$$

where $1 / 2 \leq \beta \leq 1$.

For Tsallis, we have two relations with $\alpha \neq \beta(\alpha>\beta)$ : if $\eta<1$ (from Equation (19) of [24]):

$$
2 \beta\left(\frac{\beta}{\alpha}\right)^{\frac{1}{2(\alpha-1)}} \geq \frac{\Delta \Omega_{q}^{1 / 3}}{\delta x \delta p} \geq 2 \beta\left(\frac{\beta}{\alpha}\right)^{\frac{1}{2(\alpha-1)}}\left[\frac{1}{1+(1-\alpha)\left(H_{\beta}^{x}+H_{\beta}^{p}\right)}\right]^{\alpha /(\alpha-1)}
$$

while if $\eta>1$ (from Equation (20) of [24]):

$$
2 \beta\left(\frac{\alpha}{\beta}\right)^{\frac{1}{2(1-\alpha)}} \geq \frac{\Delta \Omega_{q}^{1 / 3}}{\delta x \delta p} \geq 2 \beta\left(\frac{\alpha}{\beta}\right)^{\frac{1}{2(1-\alpha)}}\left[\frac{1}{1+(\alpha-1)\left(H_{\beta}^{x}+H_{\beta}^{p}\right)}\right]^{\alpha /(\alpha-1)}
$$

where:

$$
\eta(\alpha, \beta)=\left(\frac{\beta}{\alpha}\right)^{1 /(2 \alpha)}(2 \beta)^{(\alpha-1) / \alpha}\left(\frac{\delta x \delta p}{\Delta \Omega_{q}^{1 / 3}}\right)^{(\alpha-1) / \alpha}
$$

The distribution of momentum and position with different entropic parameters are taken with the same elementary cell $\Delta \Omega_{q}$.

The meaning of the above relations can be summarized as follows. Once the effective statistical framework (Shannon, Rényi, Tsallis or others), and, therefore, the corresponding volume of the elementary cell, is determined by the physical characteristics of the system, the only meaningful measurements of two conjugate quantities, such as position and momentum, are those whose uncertainty product satisfies the above relations.

\section{Astrophysical and Cosmological Implications}

Experiments have been arranged in astrophysics to measure the cosmic red shift, the variation of the fine-structure constant in space and time, and to observe a cosmological rate of change of the Planck constant: results are under scrutiny and do not appear to give conclusive answers. Cosmic acceleration should be verified by future ground-based laboratory experiments, where dark matter and dark energy are looked for.

It is believed that phase-space structures with a volume smaller than $h^{3}$ do not exist or do not matter; however, Zurek [28] has shown that sub-Planck structures exist in the quantum version of classically chaotic systems. This situation is allowed in non-extensive statistical mechanics [29], where an elementary cell smaller than the Planck constant can be admitted at particular values of the entropic parameters. 
A conjecture on a hierarchical cosmological time evolution of the Planck constant has been accepted. This hypothetical phenomenon might be responsible for the cosmological high redshifts as an alternative mechanism to the Doppler effect. The Bohr radius of a hydrogen atom and the probability of the alpha decay of atomic nuclei have been estimated: both results seem to be compatible with observational data concerning the light elements' abundance in the early Universe [30].

Several laboratory measurements of the variation in time of the fine-structure constant assume that the variation is entirely caused by the dependence on time of the Planck constant. Laboratory measurements are reviewed by J. P. Uzan [31].

Insofar as the microscopic world is described by the principles of quantum mechanics, physical results must follow the uncertainty principle of space coordinate and momentum and depends on the Planck constant: for instance, atomic and nuclear energy levels are functions of the fine structure constant.

On the other side, the uncertainty principle and the Planck constant are not confined to microscopic physics, but they also determine the fundamental characteristics of the macroscopic physics, including the astrophysical phenomena of large objects, cosmic radiation or the cosmological evolution of the universe.

Laboratory experiments measure the present value of the Planck constant $h$ and, indirectly, the corresponding quantum phase-space elementary cell. However, there exist recent astrophysical observations that should test the value of $h$ in the past [31-33]. These first observations suggest that the Planck constant was smaller in the past.

If this smaller Planck constant must apply also to the elementary cell of the phase space, we could describe these results by a generalized statistical mechanics caused, for instance, by non-ideal conditions in the past.

Therefore, we may justify an elementary cell or a Planck constant different (smaller) than the actual value:

- by observing the cosmological rate of change of the Planck constant, future cosmic acceleration can be verified from ground-based laboratory experiments [33];

- by observations, suggesting a smaller value of the fine structure constant at high redshift [32];

- by the classical interpretation [16-18] of the constant $h$;

- by a fractal space-time [34].

\section{Conclusions}

In this work, we have shown that for classical systems non-describable by the Maxwell-BoltzmannGibbs statistics, but by generalized statistics (like the Tsallis $q$-statistics), the number of accessible states and the elementary cell volume differ from the ideal case: the deformed phase-space elementary volume is always larger than the ideal one independently of the sign of $1-q$, because the leading correction depends on $(1-q)^{2}$, while the number of states and the elementary cell are smaller or larger, depending on $q$. In classical systems, the elementary cell is not a universal constant, and the elementary cell can have a volume slightly smaller than $h^{3}$ in spite of the uncertainty principle. 
If we assume that the volume of the elementary cell is $h^{3}$, this cell could have been smaller in the past because $h$ was smaller for its intrinsic evolution or, vice versa, one could correlate the evolution of $h$ to the change of the effective volume of the elementary cell, due to stronger spatial and temporal correlations or the fractal measure of space when densities and interactions were stronger. These interpretations are especially interesting if the need for smaller values of the Planck constant in the past to explain some astrophysical effects, de-coherence effects and electromagnetic problems is confirmed.

We have shown results only in the limit of small deviations from the standard dynamics, since they seem to be the most relevant for possible applications, but the qualitative considerations and results would be similar without these simplifying approximations.

\section{Conflicts of Interest}

The authors declare no conflict of interest.

\section{References}

1. Kaniadakis, G.; Lissia, M.; Rapisarda, A(Eds). Non extensive thermodynamics and physical applications. Physica A 2002, 305, 1-350.

2. Kaniadakis, G.; Lissia, M. (Eds). News and Expectations in Thermostatistics. Physica A 2004, $340,1-520$.

3. Tsallis, C. Possible generalization of Boltzmann-Gibbs statistics. J. Stat. Phys. 1988, 52, 479-487.

4. Tsallis, C. Introduction to Nonextensive Statistical Mechanics: Approaching a Complex World; Springer: New York, NY, USA, 2009.

5. Quarati, F.; Quarati, P. Phase space cell in nonextensive classical systems. Entropy 2003, 5, 239-251.

6. Abe, S. Essential discreteness in generalized thermostatistics with non-logarithmic entropy. $E P L$ 2010, $90,50004$.

7. Sommerfeld, A. Thermodynamics and Statistical Mechanics; Bopp, F., Meixner, J., Eds.; Academic Press: New York, NY, USA, 1964.

8. Bohm, D.; Schützer, W. The general statistical problem in physics and the theory of probability. Il Nuovo Cimento Ser. 10 1955, 2, 1004-1047.

9. Diu, B.; Guthmann, C.; Lederer, D.; Roulet, B. Physique Statistique; Hermann: Paris, France, 1989; pp. 239-241. Complement IIF.2.

10. Kaniadakis, G. Statistical mechanics in the context of special relativity. Phys. Rev. E 2002, 66, 056125.

11. Kaniadakis, G. Theoretical foundations and mathematical formalism of the power-law statistical distributions. Entropy 2013, 15, 3983-4010.

12. Gross, D.H.E. Classical equilibrium thermostatistics, "Sancta sanctorum of Statistical Mechanics" from nuclei to stars. Physica A 2004, 340, 76-84.

13. Shalizi, C.R. Tsallis Statistics, Statistical Mechanics for Non Extensive Systems and Long Range Interactions, 2012. Available online: http://http://vserver1.cscs.lsa.umich.edu/ crshalizi/ notebooks/tsallis.html (accessed on 15 December 2012). 
14. Andresen, B. Comment on "Essential discreteness in generalized thermostatistics with non-logarithmic entropy" by Abe Sumiyoshi. EPL 2010, 92, 40005.

15. Abe, S. Reply to the comment by B. Andresen. EPL 2010, 92, 40006.

16. Boyer, T.H. Recalculations of long-range van der Waals Potentials. Phys. Rev. 1969, 180, 19-24.

17. Boyer, T.H. Is Planck's Constant h a "Quantum" Constant? 2008. arXiv:0803.3612. Available online: http://arxiv.org/abs/0803.3612 (accessed on 2 April 2013).

18. Boyer, T.H. Is Planck's Constant h a "Quantum" Constant? An Alternative Classical Interpretation, 2013. arXiv:1301.6043. Available online: http://arxiv.org/ abs/1301.6043 (accessed on 2 April 2013).

19. Chung, W.S. New Exponential, Logarithm and q-probability in the Non-Extensive Statistical Physics, 2013. arXiv:1302.1865. Available online: http://arxiv.org/ abs/1302.1865 (accessed on 25 April 2013).

20. Partovi, M.H. Entropic formulation of uncertainty for quantum measurements. Phys. Rev. Lett. 1983, 50, 1883-1885.

21. Bialynicki-Birula, I. Entropic uncertainty relations. Phys. Lett. A 1984, 103, 253-254.

22. Rajakopal, A.K. The Sobolev inequality and the Tsallis entropic uncertainty relation. Phys. Lett. A 1995, 205, 32-36.

23. Bialynicki-Birula, I. Formulation of the uncertainty relations in terms of the Renyi entropies. Phys. Rev. A 2006, 74, 052101.

24. Wilk, G.; Wlodarczyk, Z. Uncertainty relations in terms of the Tsallis entropy. Phys. Rev. A 2009, 79, 062108.

25. Bialynicki-Birula, I.; Rudnicki, L. Comment on "Uncertainty Relations in Terms of the Tsallis entropy, 2010. arXiv:1001.4666. Available online: http://arxiv.org/abs/ 1001.4666 (accessed on 5 July 2013).

26. Wilk, G.; Wlodarczyk, Z. Generalized thermodynamic uncertainty relations. Physica A 2011, 390, 3566-3572.

27. Bialynicki-Birula, I.; Rudnicki, L. Entropic Uncertainty Relations in Quantum Physics. In Statistical Complexity; Sen, K.D., Ed.; Springer: Heidelberg, Germany, 2011; Chapter 1.

28. Zurek, W.H. Sub-Planck structure in phase space and its relevance for quantum decoherence. Nature 2001, 412, 712-717.

29. Wolf, C. Anomalous statistics and the rescaling of Planck's constant. Aperion 2002, 9, $26-32$.

30. Avetissian, A.K. Planck's constant variation as a cosmological evolution test for the early universe. Gravit. Cosmol. 2009, 15, 10-12.

31. Uzan, J.-P. The fundamental constants and their variation: observational and theoretical status. Rev. Mod. Phys. 2003, 75, 403-455.

32. Webb, J.K.; King, J.A.; Murphy, M.T.; Flambaum, V.V.; Carswell, R.F.; Bainbridge, M.B. Indications of a spatial variation of the fine structure constant. Phys. Rev. Lett. 2011, 107, 191101.

33. Seshavatharam, U.V.S.; Lakshminarayana, S. Is Planck's Constant A Cosmological Variable? Int. J. Astron. 2013, 2, 11-15.

34. Garcia-Morales, V.; Pellicer, J. Microcanonical foundation of nonextensivity and generalized thermostatistics based on the fractality of the phase space. Physica A 2006, 361, 161-172. 


\section{A. Appendix}

In this Appendix, we give a few more details about the expansion of $M_{q}^{\otimes_{q} N}$ for values of $q$ close to one.

From the definition of the $q$-power [19]:

$$
M^{\otimes_{q} N}=\left[N M^{(1-q)}-N+1\right]^{1 /(1-q)}
$$

we expand the logarithm of the $N$-th $q$-power of $M$ up to the leading correction in $x \equiv(1-q) \ln M$ :

$$
\begin{aligned}
\ln \left(M^{\otimes_{q} N}\right) & =\frac{\ln M}{x} \ln \left[1+N\left(e^{x}-1\right)\right]=\frac{\ln M}{x}\left[N\left(x+\frac{x^{2}}{2}\right)-\frac{(N x)^{2}}{2}+o\left(x^{3}\right)\right] \\
& =\ln M^{N}-\frac{x(N-1)}{2} \ln M^{N}+o\left(x^{2}\right)
\end{aligned}
$$

Considering the case $N \gg 1$, taking the exponential and substituting back $x=(1-q) \ln M$, the result is:

$$
M^{\otimes_{q} N}=M^{N}\left[1-\frac{x N^{2}}{2} \ln M+o\left(x^{2}\right)\right]=M^{N}\left[1+\frac{(q-1)}{2}\left(\ln M^{N}\right)^{2}+o\left((1-q)^{2}\right)\right]
$$

Note that in the opposite limit $N=1$, the first correction in $q-1$ vanishes (see Equation (48)); in fact, the case $N=1$ does not depend on $q$ :

$$
M^{\otimes_{q} 1}=\left[M^{(1-q)}\right]^{1 /(1-q)}=M
$$

Since the difference between $M_{0}$ and $M_{q}$ is of the order $(1-q)^{2}$ (see Equation (21) and consider that $\left.W=N ! M^{N}\right)$, we can take $M^{N}=M_{q}^{N}=M_{0}^{N}=\Omega_{0} /\left(\Delta \Omega_{0}\right)^{N}$ inside the logarithm and consider only terms up to $1-q$ :

$$
M^{\otimes_{q} N}=\frac{\Omega_{q}}{\left(\Delta \Omega_{q}\right)^{N}}=\frac{\Omega_{0}}{\left(\Delta \Omega_{0}\right)^{N}}\left[1+\frac{q-1}{2}\left(\ln \left(\frac{\Omega_{0}}{\left(\Delta \Omega_{0}\right)^{N}}\right)\right)^{2}\right]
$$

The expression of the number of available states becomes:

$$
W_{q}=N ! \frac{\Omega_{q}}{\left(\Delta \Omega_{q}\right)^{N}}=N ! \frac{\Omega_{0}}{\left(\Delta \Omega_{0}\right)^{N}}\left[1+\frac{q-1}{2}\left(\ln \left(\frac{\Omega_{0}}{\left(\Delta \Omega_{0}\right)^{N}}\right)\right)^{2}\right]
$$

or, in other notation:

$$
W_{q}=W_{0}\left[1+\frac{q-1}{2} N^{2}\left(\ln \left(\frac{W_{0}}{N !}\right)^{1 / N}\right)^{2}\right]=W_{0}\left[1+\frac{q-1}{2}\left(\ln \left(\frac{W_{0}}{N !}\right)\right)^{2}\right]
$$

\section{B. Appendix}

In this Appendix, as an application of the expressions reported in the main text based on the q-definition of the elementary cell, we give the expression of the entropy $S_{q}$ using the rules of $q$-algebra for $M_{q}^{\otimes_{q} N}$ :

$$
\frac{S_{q}^{(1)}}{k}=\ln _{q} w_{q} \approx \frac{\left(M_{q}^{\otimes_{q} N}\right)^{1-q}-1}{1-q}
$$


In the case of one particle:

$$
\frac{S_{q}^{(1)}}{k}=\frac{W_{0}^{1-q}-1}{1-q}
$$

Using as suggested by Sommerfeld [7] for one particle $W_{0}=3 \cdot 10^{4}$, let us give as examples:

$$
\begin{aligned}
\frac{1}{k} S_{q=1.02}^{(1)} & =9.32 \\
\frac{1}{k} S_{q=1.005}^{(1)} & =10.05 \\
\frac{1}{k} S_{q=1}^{(1)} & =\ln W_{0}=10.31
\end{aligned}
$$

we see that $S_{q}$ decreases as $q$ increases from $q=1$. In fact, for $|1-q| \ll 1 / \ln W_{0} \approx 0.1$, one finds $S_{q}^{(1)} / k \sim \ln W_{0}\left[1-(1 / 2)(q-1) \ln W_{0}\right]$.

We calculate now the $q$-entropy for $N$ identical particles using the $q$-algebra for the sum:

$$
\frac{1}{k} S_{q}(N)=\frac{1}{k} S_{q}^{\oplus_{N}}(1)=\frac{1}{1-q}\left\{\left[W_{0}\left(1+\frac{q-1}{2} \ln W_{0}\right)\right]^{(1-q) N}-1\right\}
$$

For large $N$ and $q>1$, or, better, for $N(q-1) \gg 1 / \ln W_{0}$, which means in our case $N \gg 1 /(10(q-1)$, one finds $S_{q}(N) / k \approx 1 /(q-1)\left(1-\exp \left(-(q-1) N \ln W_{0}\right)\right)$. In the opposite limit, $N|q-1| \ll 1 / \ln W_{0}$, which means in our case $(q-1) \ll 1 /(10 N)$, one finds $S_{q}(N) / k \approx N \ln W_{0}-(q-1) N \ln A(N \ln A-$ $1) / 2$. In general, the entropy grows linearly as $N \ln W_{0}$, as long as it is smaller than $1 /(q-1)$; then, it saturates to the constant $1 /(q-1)$. As numerical examples for the chosen $W_{0}=3 \cdot 10^{4}$ :

$$
\begin{array}{rlrl}
q=1.02 & \frac{1}{k} S_{q}(N) & \approx \frac{1}{q-1}=50 \\
q=1.005 & \frac{1}{k} S_{q}(N) & \approx \frac{1}{q-1}=200 \\
q=1 & \frac{1}{k} S_{q}(N)=N \ln W_{0} \approx 10.31 N
\end{array}
$$

(c) 2013 by the authors; licensee MDPI, Basel, Switzerland. This article is an open access article distributed under the terms and conditions of the Creative Commons Attribution license (http://creativecommons.org/licenses/by/3.0/). 